\title{
The Identification of Electrical Properties of Acupuncture Points for Asthma Diagnosis
}

\author{
Norienna Valendiani Risti ${ }^{1}$, Welina Ratnayanti Kawitana ${ }^{1}$, Tri Anggono Prijo ${ }^{1}$ \\ ${ }^{1}$ Department of Physics, Faculty of Science and Technology, Universitas Airlangga. \\ Article History \\ Received : September 2021, Revised : October 2021, Accepted : October 2021, Online : December 2021
}

\begin{abstract}
The aim of this research is to identify the electrical potential profile on the acupoint betwen healthy people and the patient of asthma. The raw data has taken by recording the electrical potential profile on the acupoints: Feishu, Pishu, and Shenshu from 10 healthy women and the 10 women with asthma attain the age of 20-30 years old based on the second data observation at the Local Government Clinic Kalijudan, Surabaya. Potential profile of the organs were the electrical signals form. It was achieved by the result of electrical potential which was based time recording. Recording time was done for 180 second. The results couldn't be differentiated significantly, so it needs the other signals processing with FFT analyze method with cutting as the data frames. It was done every 5 second. Based on the result of analyzing the amplitude of each frequency group, the significant differences are on the acupoint Shenshu : 0$5 \mathrm{~Hz}$ with $\mathrm{p}=0.001$, on the acupoint Phishu $148-152 \mathrm{~Hz}$ with $\mathrm{p}=0.010$, on the acupoint Feishu for frequency 198-203 $\mathrm{Hz}$ with $\mathrm{p}=0.004$ and on the acuponit Phishu $\mathrm{p}=0.011$, for frequency $348-352 \mathrm{~Hz}$ on the acupoint Feishu and Shenshu have both value is $\mathrm{p}=0.004$ and $398-402 \mathrm{~Hz}$ with $\mathrm{p}=0.009$ on the acupoint Phishu. According to the preference, it was found that the electrical potential profile on the acupoints of the healthy people has lower amplitude than the people with asthma. Then, the analyze of electrical potential profile on the acupoints can be used for asthma diagnose. Keywords : electrical biopotential, acupoint, asthma, FFT.
\end{abstract}

\section{INTRODUCTION}

Stress is triggered by bad life style and many daily activities, moreover the fatigue or inappropriate meal times. This matter impact to the person is susceptible to dangerous diseases that attack vital organs resulting in death. Some examples of dangerous diseases such as stroke, hypertension, hepatitis to lung disorders. From these types of disorders, asthma is one of the lung disorders that are often encountered in everyday life. Asthma is a disease characterized by an increased responsiveness of the trachea and bronchi to various stimuli whose manifestations are difficulty in breathing, due to a complete narrowing of the airways (Alsagaff et al, 1989). So, it takes a therapy for diagnosis or cure. The acupuncture therapy method was used for diagnosis in this study. The acupuncture therapy method which consists of acupuncture points and their meridians is a therapeutic method by providing stimulation at acupuncture points. Stimulation at these acupuncture points can record certain organs through the meridians. One of the benefits of using the diagnosis of organ function is by analyzing the electrical potential profile at acupuncture points. The organ function abnormalities have measured by utilizing the electrical activity of organs through acupuncture points to be observed and using as an indicator of organ dysfunction in accordance with research conducted by Puspa Erawati et al (2004).

In this study, specific acupuncture points were used for the back Shu meridian organs, namely the Feishu (Lung), Pishu (Spleen), and Shenshu (Kidney) points. The goal of this study is the utilization of electrical activity from organs through acupuncture points can be analyzed and then the electrical potential profile is used as an indicator of functional abnormalities of the lungs of asthmatics.

The obtained of electrical potential profile at the acupuncture points will be analyzed with the Fast Fourier Transform converting the time domain electrical potential profile signal into a frequency domain electrical potential profile through the IWorx Labscrib software. This result will indicate the healthy conditions and the patients with asthma.

By distinguish the electrical potential profiles of these two conditions, it is hoped that it become a new diagnostic method for other organs affected by asthma. The diagnostic method uses the principles of physics and can determine the implementation and importance of the principles of physics in research methods, especially signal analysis. 


\title{
LITERATURE RIVIEW
}

\begin{abstract}
Asthma
Asthma is a disease characterized by an increased responsiveness of the trachea and bronchi to various kinds of stimuli whose manifestations are difficulty breathing, due to a complete narrowing of the airways. This narrowing is dynamic and the degree of narrowing can vary, either spontaneously or because of the administration of drugs. The basic abnormality appears to be a change in the patient's immunological status.
\end{abstract}

\section{Acupuncture}

Acupuncture is in a special field among other fields of medicine, namely the field of bioelectricity. In this study, the Back Shu point is used, which is the point where the Qi of the organ radiates to the surface of the body and the point is located at the level of the organ concerned (Saputra and Idayanti, 2005). The following is the location of the back Shu acupuncture points used in this study:

1. Feishu (BL-13): is a point of Lung Shu, located between the III-IV thorax, 2 fingers lateral to the du meridian.

2. Pishu (BL-20): is the Shu Spleen point, located between the thoracic IX-XII, 2 fingers lateral to the meridian du.

3. Shenshu (BL-23): is the Shu point of the Kidney, located between the Lumbar II-III, 2 fingers lateral to the meridian du.

\section{Electrical nervous system and neurons}

Nerve cells function to receive, interpret, and transmit electrical messages.

\section{Electric potential}

On the entire surface or membrane of the neuron there is a potential difference (voltage) due to the presence of more negative ions on the inside of the membrane than on the outside.

\section{Introduction to iworx labscribe}

The data acquisition unit in the IWORX teaching kits is a four-channel recording device. Channels 1 and 2 on IWX/214 and 204 and Channel 1 on IWX/214 have made biopotential amplifiers that receive electrical input via AMMI cables. Mode controllers for these channels expect precise filter sets for recording ECG, EEG, EMG, and signals from other sources. 


\section{EXPERIMENT SET UP}

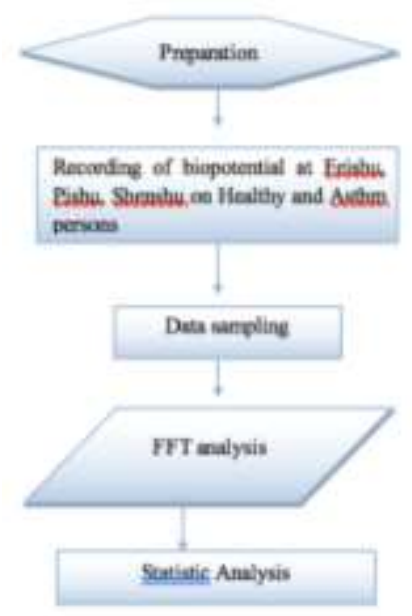

FIGURE 2. The flow chart of experiment procedure

\section{Experimental Procedure:}

1) Preparation of hardware and software for measuring and recording the electric potential profile as shown in Figure 3

2) The IWX/214 hardware and computer are turn on then connected by using a USB cable.

3) On the computer, open the Labscribe program in the start menu. Then click the tools menu in the program and click Find Hardware

4) Conecting the electrodes on the Feishu, Phishu and Shenshu points for 3 minutes (normal and suspect person)

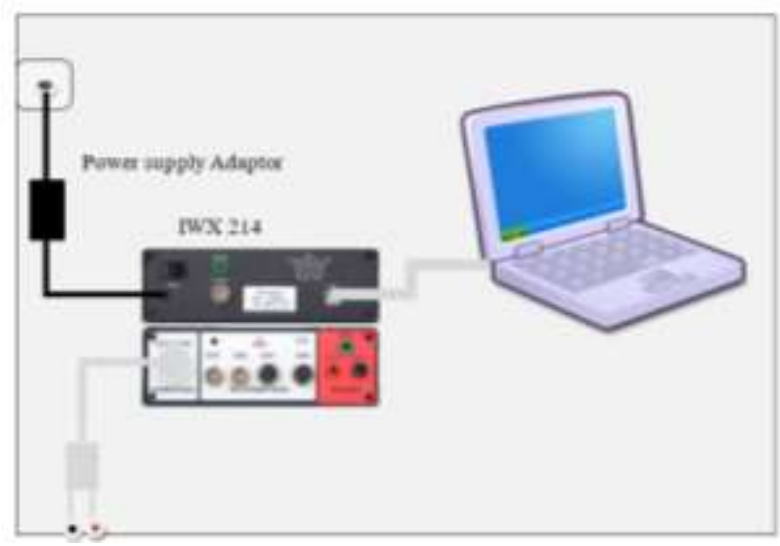

FIGURE 3. The schematic of experiment set-up 


\section{EXPERIMENT RESULT}

\section{Time domain and frequency domain electric potential profile}

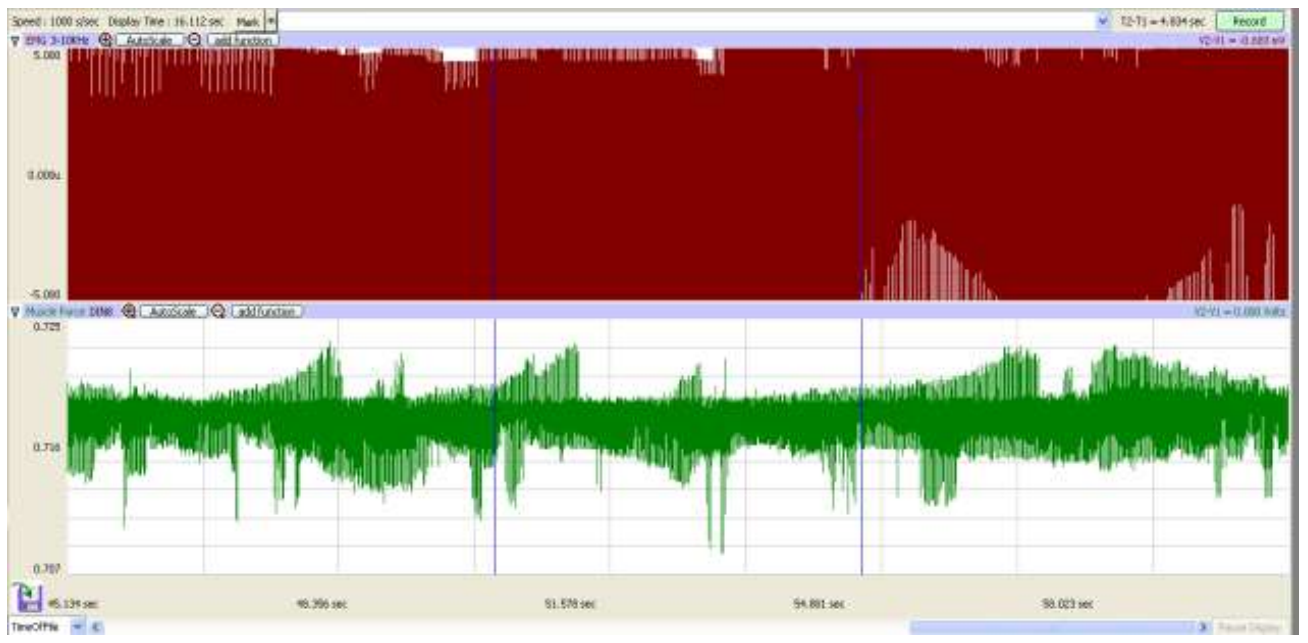

FIGURE 4. Time Domain Electrical Potential Profile in healthy people. The abscissa axis represents the data sampling time range (ms), and the ordinate axis represents the stress as a function of time (V/ms).

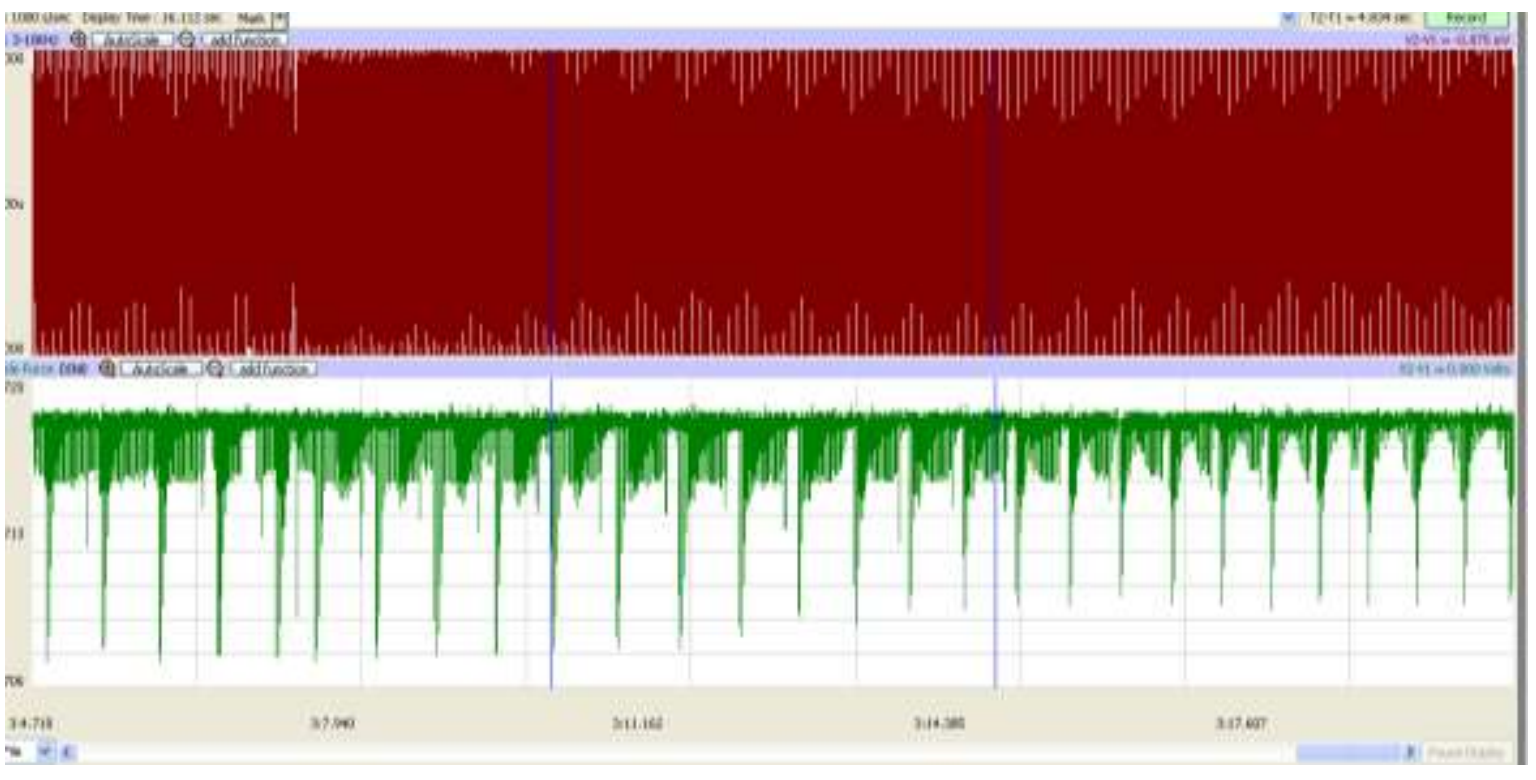

FIGURE 5. Time Domain Electrical Potential Profile in asthmatics. The abscissa represents the data sampling time range (ms), and the ordinate is the voltage as a function of time $(\mathrm{V} / \mathrm{ms})$ 


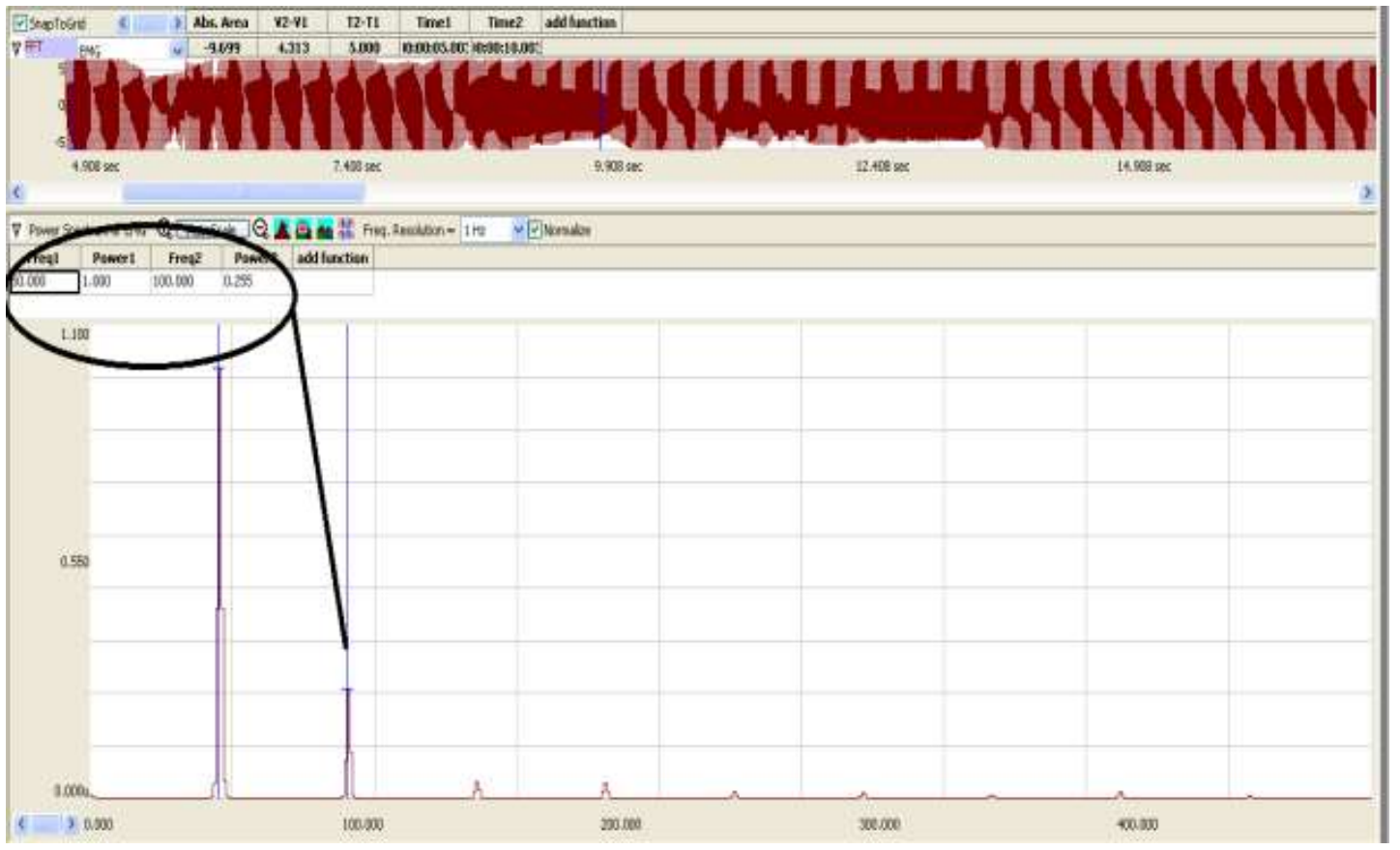

FIGURE 6. Frequency Domain Electric Potential Profile in Healthy People. The abscissa represents the frequencies that appear $(\mathrm{Hz})$, and the ordinate represents the amplitude as a function of frequency (Volt $/ \mathrm{ms})$

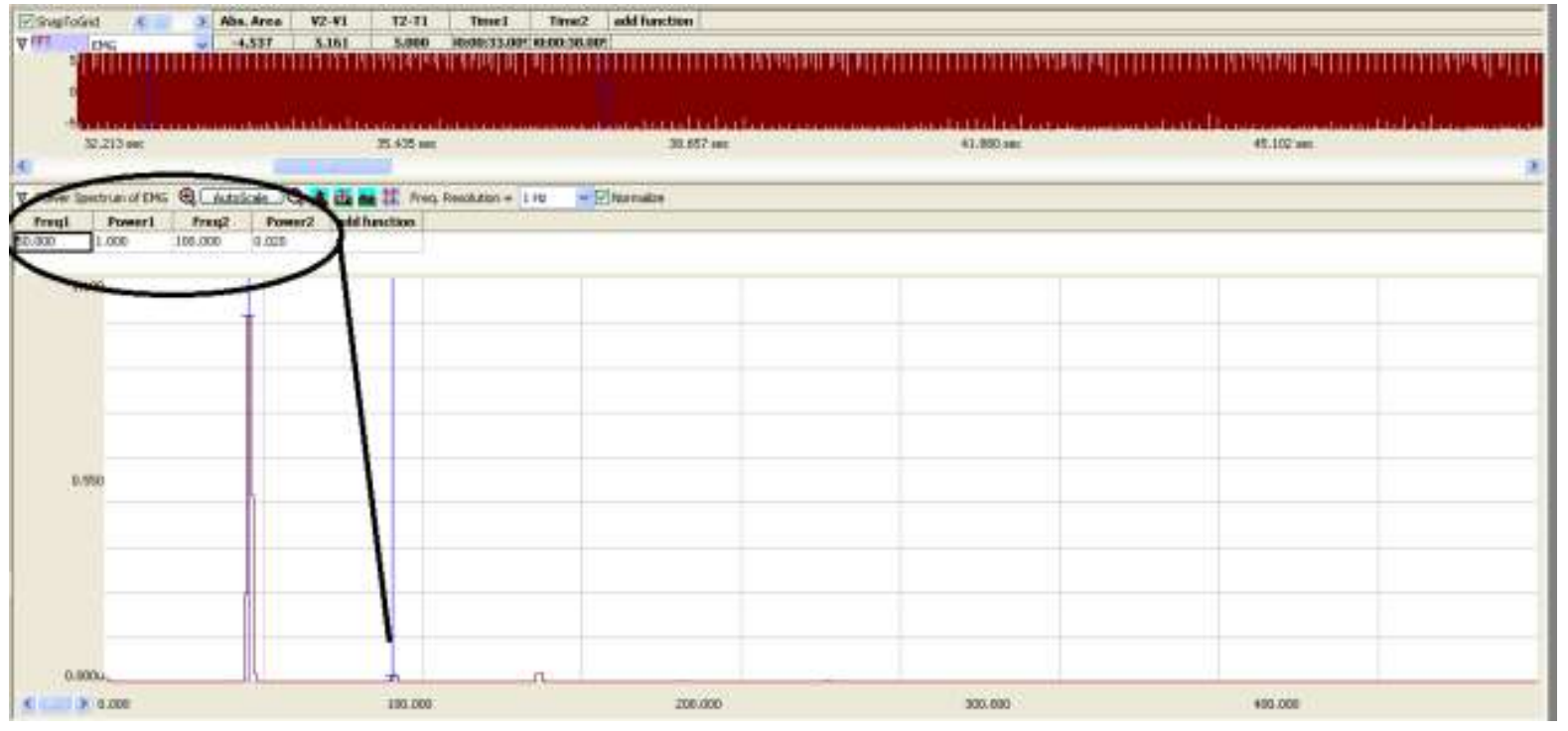

FIGURE 7. Frequency Domain Electrical Potential Profile in asthmatics. The abscissa represents the frequencies that appear $(\mathrm{Hz})$, and the ordinate represents the amplitude as a function of frequency (Volt/ms). 


\section{The result of Average Amplitude test of Each Frequency Group on Electrical Potential Profile Acupuncture Points Frequency Domain Healthy and Asthma Person}

TABLE 1. SPSS-Free Sample T Test Summary

\begin{tabular}{|c|c|c|c|c|c|}
\hline \multirow{2}{*}{$\begin{array}{l}\text { Frekuensi } \\
(\mathbf{H z})\end{array}$} & \multirow[t]{2}{*}{ Point } & \multicolumn{2}{|c|}{$\begin{array}{c}\text { Average of Amplitudo } \\
\text { (Vs) }\end{array}$} & \multirow{2}{*}{$\begin{array}{l}\text { Significant } \\
\text { Value }\end{array}$} & \multirow{2}{*}{ Description } \\
\hline & & Healthy & Asthma & & \\
\hline \multirow{3}{*}{$0-5$} & Feishu & 0.026 & 0.014 & 0.53 & No Difference \\
\hline & Phishu & 0.03 & 0.027 & 0.856 & No Difference \\
\hline & Shenshu & 0.261 & 0.022 & 0.001 & DIFFERENCE \\
\hline \multirow{3}{*}{$98-102$} & Feishu & 0.384 & 0.288 & 0.527 & No Difference \\
\hline & Phishu & 0.541 & 0.494 & 0.771 & No Difference \\
\hline & Shenshu & 0.486 & 0.491 & 0.979 & No Difference \\
\hline \multirow{3}{*}{$148-152$} & Feishu & 0.062 & 0.106 & 0.221 & No Difference \\
\hline & Phishu & 0.196 & 0.053 & 0.01 & DIFFERENCE \\
\hline & Shenshu & 0.045 & 0.065 & 0.506 & No Difference \\
\hline \multirow{3}{*}{$198-202$} & Feishu & 0.175 & 0.045 & 0.004 & DIFFERENCE \\
\hline & Phishu & 0.107 & 0.05 & 0.011 & DIFFERENCE \\
\hline & Shenshu & 0.043 & 0.081 & 0.167 & No Difference \\
\hline \multirow{3}{*}{$248-252$} & Feishu & 0.111 & 0.048 & 0.108 & No Difference \\
\hline & Phishu & 0.022 & 0.027 & 0.452 & No Difference \\
\hline & Shenshu & 0.025 & 0.028 & 0.771 & No Difference \\
\hline \multirow{3}{*}{ 298-302 } & Feishu & 0.025 & 0.027 & 0.783 & No Difference \\
\hline & Phishu & 0.024 & 0.023 & 0.956 & No Difference \\
\hline & Shenshu & 0.016 & 0.024 & 0.418 & No Difference \\
\hline \multirow{3}{*}{$348-352$} & Feishu & 0.069 & 0.024 & 0.004 & DIFFERENCE \\
\hline & Phishu & 0.025 & 0.014 & 0.082 & No Difference \\
\hline & Shenshu & 0.075 & 0.038 & 0.004 & DIFFERENCE \\
\hline \multirow{3}{*}{$398-402$} & Feishu & 0.016 & 0.02 & 0.595 & No Difference \\
\hline & Phishu & 0.026 & 0.005 & 0.009 & DIFFERENCE \\
\hline & Shenshu & 0.013 & 0.026 & 0.389 & No Difference \\
\hline
\end{tabular}

1. There is a significant difference in the $0-5 \mathrm{~Hz}$ frequency group with $\mathrm{p}=0.001$ at the Shenshu point, 148 $152 \mathrm{~Hz}$ with $\mathrm{p}=0.010$ at the Phishu point, $198-203 \mathrm{~Hz}$ with $\mathrm{p}=0.004$ at the Feishu point while the $\mathrm{p}$ value $=0.011$ at the Phishu, $348-352 \mathrm{~Hz}$ at the Feishu and Shenshu points both have the same $\mathrm{p}$ value, namely $\mathrm{p}=0.004$ and $398-402 \mathrm{~Hz}$ with $\mathrm{p}=0.009$ at the Phishu point.

2. There is no significant difference in other frequencies and at other acupuncture points.

\section{DISCUSSION}

The recording of the electrical potential profile at the acupuncture points has done in order to show the work function of the organ based on the electrical profile. By obtaining the results of different electrical profiles in healthy and asthmatic conditions, it is hoped that later it will be able to show the condition of the organs for further diagnosis purposes based on the electrical profiles from acupuncture points to organs as well as for specific therapeutic purposes to organs whose function is impaired.

The electrical potential profiles at acupuncture points has resulted from recording the electrical potential at Feishu (BL 13) acupuncture points related to the lung organ, Pishu (BL 20) related to the spleen organ, and Shenshu (BL 23) related to the kidney organ for 180 seconds. The electrical activity of organs through acupuncture 
points can be used as information for the diagnosis of organ abnormalities. The pulmonary organs focus on the kidneys and spleen. The three selected acupuncture points were acupuncture points that are in one meridian with the spleen organ near the pancreas, namely the bladder meridian (Back Shu).

The electrical potential profile is a graph of voltage as a function of time that represents the electrical activity of organs that change over time. If the time potential profile in the healthy group to be compared with the Asthma group, there was difficulties in determining the parameters as comparison criteria because of the lack of uniformity in the pattern in each group. The potential difference from the acupuncture points was the electromagnetic waves emitted by the organ. This wave was a combination of several harmonic waves of different frequencies that occur simultaneously. Healthy people who record their electrical potential are classified into the healthy group with test codes $\mathrm{n} 1$ to $\mathrm{n} 10$. Meanwhile, asthmatics who recorded their electrical potential were classified into the sick group with test codes $\mathrm{s} 1$ to s10.

The time domain electrical potential profile at acupuncture points cannot be distinguished directly, so the signal analysis process are required for this analysis. Therefore, Fast Fourier Transform analysis is needed in Labscribe software to change the electric potential profile of the frequency domain. Wave function of time as a linear combination, can be described from the basic frequencies of its manufacture through FFT analysis. The FFT generate spectrum frames that were sampled in a certain time interval. In this study, 20 spectrum frames were generated with sampling every 5 seconds. By using the two cursors provided by the FFT spectrum window, the peaks of the FFT spectrum can be seen in a row for their frequency and amplitude as shown in the toolbar menu. The dominant data in the table was the frequency group that appears with their respective amplitudes.

In this study, FFT (Fast Fourier Transform) analysis was used to analyze the profile of the time domain electric potential at acupoints. FFT analysis is a signal analysis method which is able to transform a signal from function of time into a frequency function. The electric potential profile is a superposition of waves composed of their constituent frequencies. The wave superposition is expressed in terms of the Fourier series $\mathrm{f}(\mathrm{t})$ function in Chapter II. The Fourier series for a periodic signal is expressed in a sinusoidal form. The characteristic components that build up the Fourier waveform are the constituent frequencies and other coefficients ( $\mathrm{a} 0$, an, and bn). The obtained electric potential profile is a non-periodic but continuous signal whose wave time function cannot be known. The Fourier analysis method is utilized to analyze this signal using this non-periodic signal can be made into a periodic one with a period ranging from $-\infty$ to $\infty$. Based on the frequency function, it will be known which frequencies are the frequencies that make up the wave function on the abscissa axis. The apparent amplitude on the ordinate axis is a function of the frequency $\mathrm{f}(\omega)$ resulting from the Fourier transform. The obtained amplitude is from 0 to 1 . This is because the frequency function $f(\omega)$ generated from the Fourier transform must be normalized.

After FFT signal analysis, all you need to do is recording the frequency and amplitude of each spectrum frame. The recording of frequency and amplitude have done by hovering the blue cursor at each peak in the FFT spectrum. By placing the cursor on the peak point of the FFT spectrum, below the toolbar icons there is information on the frequency and amplitude at that peak point. From a series of recording times, potential profiles were sampled every 5 seconds. The number of frames sampled is 20 spectrum frames with frequency groups appearing at points 0-5 Hz, $50 \mathrm{~Hz}, 98-102 \mathrm{~Hz}, 148-152 \mathrm{~Hz}, 198-202 \mathrm{~Hz}, 248-252 \mathrm{~Hz}, 298-302 \mathrm{~Hz}, 348-352 \mathrm{~Hz}$ and $398-402 \mathrm{~Hz}$ with amplitudes ranging from 0 to 1 . The $50 \mathrm{~Hz}$ frequency is covered with a frequency from PLN whose amplitude is very high, which is equal to 1 , so it is considered as noise or noise that cannot be avoided. With such a phenomenon, the $50 \mathrm{~Hz}$ frequency was not included in the observation data because its value was always the same in each test and at all points for healthy people and asthmatics. This frequency group is the frequency group that appears in the FFT spectrum frame. Based on the recorded data, there are inconsistencies if observed directly. The recorded amplitude for each frequency group in each frame of the FFT spectrum shows non-uniformity. However, from the data, it can be seen that there is a tendency for similarity, so that the non-uniformity seen in small orders is not too significant.

The SPSS 13.0 for Windows with T sample has utilized to analyze after FFT process. Prior to the free sample $\mathrm{T}$ test, the data were tested for normality using the non-parametric Kolmogorov-Smirnov test. The normal distribution result is obtained from the tested data, then the free sample $\mathrm{T}$ test was carried out. In a series of free sample $\mathrm{T}$ tests, there is a data homogeneity test using the Levenue test. By using the decision-making rules of the free sample t-test, ie the resulting p-value is smaller than 0.05 , it can be concluded that there is a significant difference between the two conditions. Next, from the conversion of the FFT spectrum data into a table, the results of the amplitudes of the 20 sampling frames are averaged and then entered into the data variables in the SPSS software. The data analyzed using SPSS are shown in the Appendix.

In this study, the frequency analyzed using statistical tests was only on the dominant frequency, namely the frequency that appeared strong and could affect the shape of the initial signal that had not been analyzed using FFT analysis. If there is an appearance that is not so strong, it means it is a noise. Biopotential recording software that has been set up automatically as an EMG signal recorder. The resulting EMG signal is in the order of microvolts, so amplification is needed to be shown on a computer screen. The EMG signal from the recorded body surface comes from the potential difference that occurs between two electrodes mounted on acupuncture points laterally as the entrance and exit of energy that has positive and negative directions. Between the 
acupuncture points and their electrical on the organs are connected by meridians as the path of energy flow. So that the electrical organs are observed passing through the meridians to the acupuncture points. EMG signal recording uses an Iworx device that can amplify 1000 times of the input signal so that it can be observed on a computer screen. So that it cannot be used as a method of analyzing the electrical potential profile for the diagnosis of asthma because in the results obtained there is a tendency for noise to appear which may be caused by the electrode used is a non-invasive method so it is very easy for interference to occur. For example, interference from radiation sources such as transmission, inherent signal instability (EMG signals have a random nature), unstable placement during recording or the entry of signals from other body components near the placement of electrodes that are exposed to small electrical stimuli so that they interfere with the signal from the target to be detected.

By using the Fourier transform, signals in a function of time that were previously indistinguishable can now be seen statistically significant differences. The tendency that arises in the electrical potential profile for healthy conditions with asthma conditions is that the resulting amplitude tends to be higher in asthmatic conditions. This shows that the signal processing method for data preparation is needed to determine the difference in the electric potential profile which was previously a function of time. By distinguishing the electrical potential profile at acupuncture points for the condition of healthy people and sick people, this analysis method can be used as a method of diagnosis for asthma.

The factors that caused no significant difference was found at other acupuncture points and other frequencies due to the inhomogeneous health profile of the testes and the possibility of an electric current that was not from the organ transmitted by the electrode. The recorded electric potential profile is a potential profile for the state at a certain time during recording. The inhomogeneous state of the testes due to psychological and physical factors causes an instantaneous change in the electric potential profile. Determination of the location of the electrodes at the acupuncture points that are not precise or the occurrence of electrode shifts can also be one of the causes of the loss of signals that should be recorded. The pattern of the electrical potential profile of the frequency function in healthy testes has a tendency for the dominant frequency to have a smaller amplitude than in sick testes. The frequency that appears is a representation of the electrical activity of the organ. This difference can be caused by the tendency of changes in electrical activity in sick people and healthy people, where the organs in sick people do more activities to balance the condition of the body.

This excess energy can be considered as a signal emitted by the organ. From the results of the study, it was found that there was a significant difference at the Shenshu point (related to the kidney organ) at low frequencies, namely $0-5 \mathrm{~Hz}$, while at high frequencies, namely 198-202 Hz, but the significant value was low at the Phishu point.

In this study, the exact reaseon of this trend is not known yet. Further and more serious research is needed to involve experts in the fields of conventional medicine and acupuncture to explore the things that happen to the organs observed in this study. However, what can be observed is that the organs that are connected to these acupuncture points are organs that are susceptible to being disturbed or prone to asthma.

\section{ACKNOWLEDGEMENT}

Department of Physics, Faculty of Science and Technology, Universitas Airlangga Surabaya as a place and provider of research facilities. Kalijudan Public Health Center as a place for examination of research tests.

\section{REFERENCE}

1. Alsagaff, Hood; Amin, Muhammad; Saleh, WBM Taib, 1989, Pengantar Ilmu Penyakit Paru, Penerbit : Airlangga University Press.

2. Ashari dan Santosa, B. P., 2005, Analisis Statistik dengan Microsoft Excell \& SPSS, Penerbit ANDI, Yogyakarta

3. Boas, Mary L., 1983, Mathematical Methods in the Physical Sciences Second Edition, John Wiley \& Son, Inc, Canada

4. Erawati, P., Astuti, S. D., dan Prijo, T. A., 2003, Analisis Profil Potensial Untuk Kelainan Fungsional Organ, Lembaga Penelitian Universitas Airlangga, Surabaya

5. Cameron, J.R, 1978, Fisika Tubuh Manusia, Diterjemahkan Oleh Brahm U. Pendit, Edisi 2, Penerbit Buku Kedokteran EGC, Jakarta

6. Carlo J, Deluca, 1976, The use of Surface EMG in Biomechanics,

7. Saputra, K., Idayanti, A., 2005, Akupunktur Dasar, Airlangga University Press, Surabaya

8. Widhiarso, Wahyu, Cara Membaca SPSS, Fakultas Psikologi UGM, Yogyakarta

9. Wijayanto, Y. Nur. dan Hastuti, D., 2006, Rangkaian Bioamplifier untuk Mendeteksi Sifat Elektris Otot, Jurnal Elektronika No. 2 Juli-Desember 2006, Volume 6 